OPEN ACCESS

Edited by:

Anthony Baughn,

University of Minnesota, USA

Reviewed by:

Sven Hammerschmidt,

University of Greifswald, Germany

Justin A. Thornton,

Mississippi State University, USA

${ }^{*}$ Correspondence:

Oscar P. Kuipers

o.p.kuipers@rug.nl

Received: 04 January 2017 Accepted: 24 February 2017 Published: 09 March 2017

Citation:

Afzal M, Kuipers OP and Shafeeq S

(2017) Niacin-mediated Gene

Expression and Role of NiaR as a Transcriptional Repressor of niaX nadC, and pnuC in Streptococcus pneumoniae.

Front. Cell. Infect. Microbiol. 7:70 doi: 10.3389/fcimb.2017.00070

\section{Niacin-mediated Gene Expression and Role of NiaR as a Transcriptional Repressor of niaX, nadC, and pnuC in Streptococcus pneumoniae}

\author{
Muhammad Afzal ${ }^{1,2}$, Oscar P. Kuipers ${ }^{1 *}$ and Sulman Shafeeq ${ }^{3}$ \\ ${ }^{1}$ Department of Molecular Genetics, Groningen Biomolecular Sciences and Biotechnology Institute, University of Groningen, \\ Groningen, Netherlands, ${ }^{2}$ Department of Bioinformatics and Biotechnology, Government College University, Faisalabad, \\ Pakistan, ${ }^{3}$ Department of Microbiology, Tumor and Cell Biology, Karolinska Institutet, Stockholm, Sweden
}

NAD (Nicotinamide Adenine Dinucleotide) biosynthesis is vital for bacterial physiology and plays an important role in cellular metabolism. A naturally occurring vitamin B complex, niacin (nicotinic acid), is a precursor of coenzymes NAD and NADP. Here, we study the impact of niacin on global gene expression of Streptococcus pneumoniae D39 and elucidate the role of NiaR as a transcriptional regulator of niaX, nadC, and pnuC. Transcriptome comparison of the D39 wild-type grown in chemically defined medium (CDM) with 0 to $10 \mathrm{mM}$ niacin revealed elevated expression of various genes, including niaX, nadC, pnuC, fba, rex, gapN, pncB, gap, adhE, and adhB2 that are putatively involved in the transport and utilization of niacin. Niacin-dependent expression of these genes is confirmed by promoter lacZ-fusion studies. Moreover, the role of transcriptional regulator $\mathrm{NiaR}$ in the regulation of these genes is explored by DNA microarray analysis. Our transcriptomic comparison of D39 $\Delta$ niaR to D39 wild-type revealed that the transcriptional regulator $\mathrm{NiaR}$ acts as a transcriptional repressor of niaX, pnuC, and nadC. NiaR-dependent regulation of niaX, nadC, and pnuC is further confirmed by promoter lacZ-fusion studies. The putative operator site of NiaR (5'-TACWRGTGTMTWKACASYTRWAW-3') in the promoter regions of niaX, nadC, and pnuC is predicted and further confirmed by promoter mutational experiments.

Keywords: niacin, NiaR, Pneumococcus, niaX, nadC, pnuC

\section{INTRODUCTION}

Bacteria can trigger transcriptional and phenotypic programs to synchronize an adaptive response in reaction to environmental fluctuations or stresses (Edwards et al., 2013). This not only relies on the number of virulence factors it possesses, but also on the proper use of nutrients available in the human niches (Phillips et al., 1990; Titgemeyer and Hillen, 2002). A number of important vitamins and co-factors are required by bacteria to survive and grow successfully. Streptococcus pneumoniae, a major Gram-positive human pathogen and nasopharyngeal colonizer, encounters different environmental factors and has to fine-tune its gene expression accordingly (Bogaert et al., 2004; Kadioglu et al., 2008). 
Niacin (nicotinic acid), a naturally occurring vitamin B complex, is a precursor of coenzymes NAD and NADP, and plays an important role in electron transfer during metabolic processes (Wei et al., 2014). Niacin has long been used for the treatment of lipid disorders and cardiovascular disease (Wei et al., 2014). It can regulate the activity of microbial two-component systems and, subsequently, modulate the genes and phenotypes that are controlled by these regulatory proteins (McPheat et al., 1983). Particularly, niacin has been reported to repress the expression of many genes including virulence factors in Bordetella pertussis, such as pertussis toxin, adenylate cyclase toxin, and filamentous hemagglutinin (Schneider and Parker, 1982; McPheat et al., 1983; Cotter and DiRita, 2000; Cummings et al., 2006). Furthermore, the two-component system BvgA/BvgS, which is known to have a role in the regulation of virulence and colonization, becomes inactive in $B$. pertussis when niacin is present in the medium (Miller et al., 1989). Similarly, the Escherichia coli EvgA/EvgS system that confers multidrug resistance and acid tolerance is regulated by niacin (Masuda and Church, 2002, 2003; Eguchi et al., 2003; Nishino et al., 2003). Both the BvgA/BvgS system of $B$. pertussis and the EvgA/EvgS system of E. coli are part of a family of proteins that utilize a multistep phosphor-relay to trigger their responsive pathways.

It has been proposed that in S. pneumoniae niacin enters the cell through NiaX and is converted to nicotinate (nicotinic acid)-mononucleotide by PncB (Johnson et al., 2015). Nicotinate mononucleotide is then converted to nicotinic acid adenine dinucleotide by $\mathrm{NadD}$, whereafter NadE converts nicotinic acid adenine dinucleotide to nicotine adenine dinucleotide (NAD) (Johnson et al., 2015). Another important enzyme glyceraldehyde-3-phosphate dehydrogenase (GAP) is a highly conserved and a multifunctional protein with significant activity in several fundamental cell pathways (Sirover, 2011). Usually, the dehydrogenase reactions of metabolic pathways have been deemed the major sources of NADPH. Nevertheless, the importance of transhydrogenases, glucose dehydrogenases, and non-phosphorylating glyceraldehyde 3- phosphate dehydrogenase (GAPN), is becoming eminent, suggesting that the traditional view is over-simplistic (Sauer U. et al., 2004; Matsubara et al., 2011; Bräsen et al., 2014). As NAD is a vital cofactor used by all living organisms, all bacterial species make use of the pathways to reduce NAD ${ }^{+}$to NADH (Jurtshuk, 1996). $\mathrm{NAD}^{+}$is also used by bacteria as a substrate for dehydrogenases involved in breaking down aldehydes and alcohols (Nobelmann and Lengeler, 1996; Kotrbova-Kozak et al., 2007; Luong et al., 2015). Furthermore, several cellular processes in bacterial and mammalian cells also use NAD, for instance DNA ligation and repair, redox recycling in the pyruvate dehydrogenase pathway, and synthesis of acetyl-CoA for the tricarboxylic acid cycle (Ishino et al., 1986; Satoh and Lindahl, 1992; Wilkinson et al., 2001; Chalkiadaki and Guarente, 2012; Chiarugi et al., 2012; Patel et al., 2014).

YrxA (NiaR) was found to be a niacin-responsive repressor of NAD de novo synthesis in Bacillus subtilis and transcriptional regulation of NAD biosynthesis in bacteria having orthologs of $B$. subtilis $y r x A$ was determined using a comparative genomic approach and expression studies (Rodionov et al., 2008a). NiaR family members are generally conserved in the Bacillus/Clostridium group and in the unrelated Fusobacteria and Thermotogales lineages (Rodionov et al., 2008a). The NiaR regulon is not limited to the transcriptional regulation of the $n a d A B C$ but in some species it also covers niacin salvage (the $p n c A B$ genes) and contains uncharacterized membrane proteins putatively involved in niacin transport (Rodionov et al., 2008a). Moreover, members of the NiaP family (involved in niacin uptake) are not only conserved in bacteria but also in multicellular eukaryotes, including humans, suggesting the putative involvement of $\mathrm{NiaP}$ in niacin utilization in these organisms (Rodionov et al., 2008a).

This study explains the transcriptomic response of $S$. pneumoniae D39 to niacin and regulation of niaX, pnuC, and nadC genes. We established that the transcriptional regulator NiaR acts as a transcriptional repressor for niaX, pnuC, and nadC genes involved in niacin uptake and utilization. The putative operator site (5'-TACWRGTGTMTWKACASYTRWAW-3' where $\mathrm{R}=\mathrm{A} / \mathrm{G}, \mathrm{K}=\mathrm{G} / \mathrm{T}, \mathrm{S}=\mathrm{G} / \mathrm{C}, \mathrm{Y}=\mathrm{T} / \mathrm{C}, \mathrm{W}=\mathrm{A} / \mathrm{T}$ and $\mathrm{M}=$ $\mathrm{A} / \mathrm{C}$ ) of NiaR in the promoter regions of niaX, pnuC, and nadC is predicted, and subsequently confirmed by mutating NiaR operator sites in the respective promoters.

\section{MATERIALS AND METHODS}

\section{Bacterial Strains and Growth Conditions}

Bacterial strains and plasmids used in this study are listed in Table 1. S. pneumoniae D39 was grown as described previously (Kloosterman et al., 2006; Afzal et al., 2014). For $\beta$-galactosidase assays, derivatives of S. pneumoniae D39 were grown in chemically defined medium (CDM) (Neves et al., 2002) with or without $10 \mathrm{mM}$ niacin. CDM was prepared without niacin. For selection on antibiotics, media were supplemented with the following concentrations of antibiotics: $150 \mu \mathrm{g} / \mathrm{ml}$ spectinomycin and $2.5 \mu \mathrm{g} / \mathrm{ml}$ tetracycline for S. pneumoniae, and $100 \mu \mathrm{g} / \mathrm{ml}$ ampicillin for E. coli. All bacterial strains used in this study were stored in $10 \%(\mathrm{v} / \mathrm{v})$ glycerol at $-80^{\circ} \mathrm{C}$. For PCR amplification, chromosomal DNA of S. pneumoniae D39 (Lanie et al., 2007) was used as a template. Primers used in this study are based on the sequence of the $S$. pneumoniae D39 genome and listed in Table 2.

\section{Construction of a niaR Mutant}

A niaR mutant (MA1300) was constructed in S. pneumoniae D39 by allelic replacement with a spectinomycin-resistance cassette. Primer pairs niaR-1/niaR-2 and niaR-3/niaR-4 were used to generate PCR fragments of the left and right flanking regions of niaR using Phusion ${ }^{\circledR}$ High-Fidelity DNA polymerase. PCR products of left and right flanking regions of niaR contain AscI and NotI sites, respectively. The spectinomycin-resistance marker, which was amplified by primers SpecR/SpecF from pORI38, also contains AscI and NotI sites on its ends. Then, by restriction and ligation, the left and right flanking regions of niaR were fused to the spectinomycin-resistance gene. The resulting ligation products were transformed to S. pneumoniae D39 wild-type and selection of the mutant was done on the appropriate concentration 
of spectinomycin. Deletion of niaR was further verified by PCR using primer pair NiaR-Conf-1/NiaR-Conf-2 and DNA sequencing.

\section{Construction of Promoter lacZ-fusions and Their Use in $\beta$-Galactosidase Assays}

Chromosomal transcriptional lacZ-fusions to niaX, pnuC, and nadC promoters were constructed in pPP2 (Halfmann et al., 2007) with primer pairs mentioned in Table 2, resulting in pMA1301-03, respectively. These constructs were further introduced into D39 wild-type and D39 $\Delta$ niaR (MA1300) resulting in strains MA1301-03 and MA1304-06, respectively. The following lacZ-fusions of $\mathrm{P} n i a X, \mathrm{P} p n u C$, and $\mathrm{P} n a d C$ with mutations in the NiaR site were made in pPP2 (Halfmann et al., 2007) using the primer pairs mentioned in Table 2: PniaX-M (mutation in the niaR site), РpnuC-M (mutation in the niaR site), PnadC-R1 (mutation in the niaR site 1), and PnadC-R2 (mutation in the niaR site 2), resulting in plasmids pMA1304-07, respectively. These constructs were introduced into the S. pneumoniae D39 wild-type strain, resulting in strains MA1307-1310, respectively. Similarly, chromosomal transcriptional lacZ-fusions to $f b a, r e x, g a p N, p n c B, g a p, a d h E$, and $a d h B 2$ promoters were constructed in pPP2 (Halfmann et al., 2007) with primer pairs mentioned in Table 2, resulting in pMA1308-14, respectively. These constructs were further introduced into D39 wild-type resulting in strains MA131117, respectively. All plasmid constructs were further checked for the presence of the right insert by PCR and DNA sequencing.

$\beta$-galactosidase assays were performed as described before (Israelsen et al., 1995; Halfmann et al., 2007) using cells that were harvested in the mid-exponential growth phase, and grown in CDM (Neves et al., 2002) with or without niacin as mentioned in the results section.

\section{Microarray Analysis}

Microarray analysis was performed as described before (Afzal et al., 2015; Shafeeq et al., 2015). For DNA microarray analysis of $S$. pneumoniae in the presence of niacin, the transcriptome of S. pneumoniae D39 wild-type grown in replicates in CDM with $10 \mathrm{mM}$ niacin was compared to that grown in CDM with $0 \mathrm{mM}$ niacin and harvested at their respective mid-exponential growth phases.

For DNA microarray analysis of D39 $\Delta n i a R$, the transcriptome of $S$. pneumoniae D39 $\Delta$ niaR was compared to $S$. pneumoniae D39 wild-type grown in replicates in complete CDM and harvested at respective mid-exponential growth phases. Complete CDM contains $8 \mu \mathrm{M}$ of niacin. The procedures for DNA microarray analysis were performed as described previously (Afzal et al., 2015; Shafeeq et al., 2015). For the identification of differentially expressed genes, a Bayesian $p<0.001$ and a fold-change cut-off $>1.5$ was applied. Microarray data have been submitted to GEO (Gene Expression Omnibus) under accession numbers GSE94511 and GSE94513.
TABLE 1 | List of strains and plasmids used in this study.

\begin{tabular}{|c|c|c|}
\hline Strain/plasmid & Description & Source \\
\hline \multicolumn{3}{|c|}{ S. PNEUMONIAE } \\
\hline D39 & Serotype 2 strain. cps 2 & Laboratory of P. Hermans. \\
\hline MA1300 & D39 $\Delta$ niaR; Spec ${ }^{R}$ & This study \\
\hline MA1301 & D39 $\Delta$ bgaA:: PniaX-lacZ; Tet $^{R}$ & This study \\
\hline MA1302 & D39 $\Delta$ bgaA:: PpnuC-lacZ; Tet ${ }^{R}$ & This study \\
\hline MA1303 & D39 $\Delta$ bgaA:: PnadC-lacZ; Tet $^{R}$ & This study \\
\hline MA1304 & MA1300 $\Delta$ bgaA:: PniaX-lacZ; Tet ${ }^{R}$ & This study \\
\hline MA1305 & $\begin{array}{l}\text { MA1300 } \Delta \text { bgaA:: PpnuC-lacZ; } \\
\text { Tet }^{R}\end{array}$ & This study \\
\hline MA1306 & $\begin{array}{l}\text { MA1300 } \Delta \text { bgaA:: PnadC-lacZ; } \\
\text { Tet }^{R}\end{array}$ & This study \\
\hline MA1307 & D39 $\Delta$ bgaA:: PniaX-M-lacZ; Tet ${ }^{R}$ & This study \\
\hline MA1308 & D39 $\Delta$ bgaA:: PnuC-M-lacZ; Tet ${ }^{R}$ & This study \\
\hline MA1309 & $\begin{array}{l}\text { D39 } \Delta b g a A:: \text { PnadC-R1-M-lacZ; } \\
\text { Tet }^{R}\end{array}$ & This study \\
\hline MA1310 & $\begin{array}{l}\text { D39 } \Delta b g a A:: \text { PnadC-R2-M-lacZ; } \\
\text { Tet }^{R}\end{array}$ & This study \\
\hline MA1311 & D39 $\Delta$ bgaA:: Pfba-lacZ; Tet ${ }^{R}$ & This study \\
\hline MA1312 & D39 $\Delta$ bgaA:: Prex-lacZ; Tet ${ }^{R}$ & This study \\
\hline MA1313 & D39 $\Delta$ bgaA:: PgapN-lacZ; Tet $^{R}$ & This study \\
\hline MA1314 & D39 $\Delta$ bgaA:: PpncB-lacZ; Tet $R$ & This study \\
\hline MA1315 & D39 $\Delta$ bgaA:: Pgap-lacZ; Tet $^{R}$ & This study \\
\hline MA1316 & D39 $\Delta$ bgaA:: PadhE-lacZ; Tet ${ }^{R}$ & This study \\
\hline MA1317 & D39 $\Delta$ bgaA:: PadhB2-lacZ; Tet ${ }^{R}$ & This study \\
\hline \multicolumn{3}{|l|}{ E. COLI } \\
\hline EC1000 & $\begin{array}{l}\mathrm{Km}^{\mathrm{R}} ; \mathrm{MC1000} \text { derivative carrying } \\
\text { a single copy of the } \mathrm{pWV} 1 \text { repA } \\
\text { gene in } \mathrm{glgB}\end{array}$ & Laboratory collection \\
\hline \multicolumn{3}{|l|}{ PLASMIDS } \\
\hline pPP2 & $\begin{array}{l}\text { Amp }{ }^{R} \text { Tet }^{R} \text {; promoter-less lacZ. } \\
\text { For replacement of bgaA with } \\
\text { promoter lacZ fusion. Derivative of } \\
\text { pPP1 }\end{array}$ & Halfmann et al., 2007 \\
\hline pMA1301 & pPP2 PniaX-lacZ & This study \\
\hline pMA1302 & pPP2 PpnuC-lacZ & This study \\
\hline pMA1303 & pPP2 PnadC-lacZ & This study \\
\hline pMA1304 & pPP2 PniaX-M-lacZ & This study \\
\hline pMA1305 & pPP2 PnuC-M-lacZ & This study \\
\hline pMA1306 & pPP2 PnadC-R1-M-lacZ & This study \\
\hline pMA1307 & pPP2 PnadC-R1-M-lacZ & This study \\
\hline pMA1308 & pPP2 Pfba-lacZ & This study \\
\hline pMA1309 & pPP2 Prex-lacZ & This study \\
\hline pMA1310 & pPP2 PgapN-lacZ & This study \\
\hline pMA1311 & pPP2 PpncB-lacZ & This study \\
\hline pMA1312 & pPP2 Pgap-lacZ & This study \\
\hline pMA1313 & pPP2 PadhE-lacZ & This study \\
\hline pMA1314 & pPP2 PadhB2-lacZ & This study \\
\hline
\end{tabular}

\section{RESULTS}

\section{Niacin-dependent Gene Regulation in S. pneumoniae D39}

Microarray comparison of S. pneumoniae D39 grown in CDM with $0 \mathrm{mM}$ to same strain grown in CDM with $10 \mathrm{mM}$ niacin was performed to explore the impact of niacin on 
TABLE 2 | List of primers used in this study.

\begin{tabular}{|c|c|c|}
\hline Name & Nucleotide Sequence $\left(5^{\prime} \rightarrow 3^{\prime}\right)$ & Restriction site* $^{*}$ \\
\hline niaX-F & CATGGAATTCTCAAACCTGAAGGTGGAGAT & ECORI \\
\hline niaX-R & CATGGGATCCGCATAACAATTGGAATCAAAATCG & BamHI \\
\hline pnuC-F & CATGGAATTCCCATATGATTCTTTCTAATGAGTTG & EcoRl \\
\hline pnuC-R & CATGGGATCCGCAAATAAGTATGCATCATTTCTCC & BamHI \\
\hline nadC-F & CATGGAATTCCCAATGGCTAGAGCAATGGC & EcoRl \\
\hline pnuC-M-F & CATGGAATTCCATGATITCTAAAATITACTACAAAGACGGTTGAC & ECORI \\
\hline nadC-R1-M-F & CATGGAATTCGACTATTATACACAAAAAAAATACAATTACCTTGACCATTGTA & ECORI \\
\hline nadC-R2-M-F & CATGGAATTCTACACAAAAAAAATACAATTGTCTTGACAATTACATTGACCCTTGTT & EcoRl \\
\hline NiaR-1 & GCCATGTTCTTGTCGCCC & - \\
\hline NiaR-2 & GCATAGGCGCGCCCAAGAGTTGGAGCAGGGC & Ascl \\
\hline $\mathrm{fba}-\mathrm{R}$ & CATGGGATCCGCATAACCGTTGTCACGGG & BamHI \\
\hline rex-F & CATGGAATTCCCTCATGGATAGCTTGGTAG & ECORI \\
\hline rex-R & CATGGGATCCGCTGTAGCTITTGGAATAGC & BamHI \\
\hline gapN-F & CATGGAATTCGGTTTGGCTGTCCCCAACC & ECORI \\
\hline gapN-R & CATGGGATCCGTCATGGCTGGAACTGTACC & BamHI \\
\hline pncB-F & CATGGAATTCGCTATGGCGAATGGGCTC & ECORI \\
\hline pncB-R & CATGGGATCCCTGGTACAAGTCCGTGTGC & BamHI \\
\hline gap-F & CATGGAATTCCGTTACGCTATGAATAATAAGGG & EcoRl \\
\hline gap-R & CATGGGATCCCGACCGATACGTCCGAAACC & BamHI \\
\hline adhE-F & CATGGAATTCGCGCTTACCTGTAAATCCC & EcoRl \\
\hline adhE-R & CATGGGATCCGAACCAACTCATCTACGTGC & BamHI \\
\hline
\end{tabular}

${ }^{\star}$ Restriction sites are underlined.

the transcriptome of S. pneumoniae D39 wild-type. CDM was prepared without niacin. A number of genes/operons were differentially expressed under our tested conditions (Table 3). A particular gene cluster (spd-0093-0095) was significantly upregulated in the absence of niacin. This gene cluster codes for three hypothetical proteins, which are putative membrane proteins. Another gene cluster (spd-1798-1802) was significantly upregulated in the absence of niacin. This gene cluster consists of a DNA-binding response regulator (encoded by spd-1798), a sensor histidine kinase (encoded by $s p d-1799$ ), two hypothetical proteins (encoded by $s p d$ 1800 and spd-1802) and an ABC transporter (encoded by spd-1801). Some genes that appear to be a part of a gene cluster were also downregulated under our tested conditions (spd-0113-15 and spd-0122-24). All of these genes code for hypothetical proteins and the role of these genes warrants further investigation.
Putative niacin biosynthesis pathway genes were significantly upregulated in the absence of niacin ( $f b a$, rex, gapN, niaX, pncB-nadE, pnuC, gap, spd-1824, nadC, adhE, and adhB2). fba codes for a fructose-bisphosphate aldolase, whereas rex encodes a redox-sensitive transcriptional regulator. Similarly, gapN encodes a glyceraldehyde-3-phosphate dehydrogenase that is involved in generation of NADPH from NADH. $p n c B$ encodes a nicotinate phosphoribosyltransferase that converts nicotinate into nicotinate D-ribonucleotide and vice versa, whereas nadE encodes a $\mathrm{NAD}^{+}$synthetase that converts deamino- $\mathrm{NAD}^{+}$to $\mathrm{NAD}^{+}$and $a d h E$ codes for an alcohol dehydrogenase. gap encodes another glyceraldehyde-3-phosphate dehydrogenase and adhE codes for an iron-containing alcohol dehydrogenase, whereas adhB2 encodes a zinc-containing alcohol dehydrogenase. NiaX (encoded by niaX) is a substrate-specific component predicted niacin ECF transporter, whereas PnuC (encoded by 
TABLE 3 | Summary of the transcriptome comparison of S. pneumoniae D39 wild-type grown in CDM with $0 \mathrm{mM}$ niacin to grown in CDM with $10 \mathrm{mM}$ niacin.

\begin{tabular}{|c|c|c|}
\hline D39 tag $^{a}$ & Function $^{b}$ & Ratioc $^{c}$ \\
\hline \multicolumn{3}{|c|}{ UPREGULATED GENES } \\
\hline spd_0093 & Hypothetical protein & 3.1 \\
\hline spd_0094 & Hypothetical protein & 2.8 \\
\hline spd_0095 & Hypothetical protein & 2.4 \\
\hline spd_0474 & Hypothetical protein & 4.6 \\
\hline spd_0475 & $\begin{array}{l}\text { CAAX amino terminal protease family } \\
\text { protein }\end{array}$ & 3.5 \\
\hline spd_0526 & $\begin{array}{l}\text { Fructose-1,6-bisphosphate aldolase, } \\
\text { class II, Fba }\end{array}$ & 1.5 \\
\hline spd_0976 & $\begin{array}{l}\text { Redox-sensitive transcriptional } \\
\text { regulator Rex }\end{array}$ & 1.5 \\
\hline spd_1004 & $\begin{array}{l}\text { Glyceraldehyde-3-phosphate } \\
\text { dehydrogenase, NADP-dependent, } \\
\text { GapN }\end{array}$ & 3.5 \\
\hline spd_1091 & $\begin{array}{l}\text { Substrate-specific component } \\
\text { predicted niacin ECF transporter, NiaX }\end{array}$ & 1.8 \\
\hline spd_1250 & $\mathrm{NAD}^{+}$synthetase, NadE & 1.5 \\
\hline spd_1251 & $\begin{array}{l}\text { Nicotinate phosphoribosyltransferase, } \\
\text { putative, PncB }\end{array}$ & 1.9 \\
\hline spd_1640 & $\begin{array}{l}\text { Ribosyl nicotinamide transporter, } \\
\text { PnuC-like, PnuC }\end{array}$ & 4.2 \\
\hline spd_1798 & DNA-binding response regulator & 2.1 \\
\hline spd_1799 & Sensor histidine kinase, putative & 2.0 \\
\hline spd_1800 & Hypothetical protein & 2.4 \\
\hline spd_1801 & ABC transporter, ATP-binding protein & 2.0 \\
\hline spd_1802 & Hypothetical protein & 2.2 \\
\hline spd_1823 & $\begin{array}{l}\text { Glyceraldehyde-3-phosphate } \\
\text { dehydrogenase, type I, Gap }\end{array}$ & 1.7 \\
\hline spd_1824 & Hypothetical protein & 2.2 \\
\hline spd_1826 & $\begin{array}{l}\text { Nicotinate-nucleotide } \\
\text { pyrophosphorylase, NadC }\end{array}$ & 4.4 \\
\hline spd_1827 & Hypothetical protein & 3.1 \\
\hline spd_1833 & PTS system, IIA component & 1.7 \\
\hline spd_1834 & $\begin{array}{l}\text { Alcohol dehydrogenase, } \\
\text { iron-containing, AdhE }\end{array}$ & 5.8 \\
\hline spd_1865 & $\begin{array}{l}\text { Alcohol dehydrogenase, } \\
\text { zinc-containing, AdhB2 }\end{array}$ & 1.7 \\
\hline spd_1874 & LysM domain protein & 3.7 \\
\hline \multicolumn{3}{|c|}{ DOWNREGULATED GENES } \\
\hline spd_0113 & Hypothetical protein & -2.9 \\
\hline spd_0114 & Hypothetical protein & -3.1 \\
\hline spd_0115 & Hypothetical protein & -2.7 \\
\hline spd_0122 & Hypothetical protein & -2.2 \\
\hline spd_0123 & Hypothetical protein & -2.4 \\
\hline spd_0124 & Hypothetical protein & -2.0 \\
\hline
\end{tabular}

a Gene numbers refer to D39 locus tags.

${ }^{b}$ D39 annotation/TIGR4 annotation (Lanie et al., 2007).

${ }^{c}$ Ratio represents the fold increase/decrease in the expression of genes in CDM with 0 $\mathrm{mM}$ Niacin to CDM with $10 \mathrm{mM}$ Niacin. Errors in the ratios never exceeded $10 \%$ of the given values.

pnuC) is a ribosyl nicotinamide transporter. NadC (encoded by $n a d C$ ) is a nicotinate-nucleotide pyrophosphorylase and has been proposed to convert quinolinate formed
TABLE 4 | Summary of transcriptome comparison of S. pneumoniae D39 $\Delta$ niaR compared to the D39 wild-type grown in complete CDM.

\begin{tabular}{llc}
\hline D39 tag $^{\mathbf{a}}$ & Function $^{\mathbf{b}}$ & Ratio $^{\mathbf{c}}$ \\
\hline spd_1091 & $\begin{array}{l}\text { Substrate-specific component predicted niacin } \\
\text { ECF transporter, NiaX }\end{array}$ & 2.1 \\
spd_1093 & $\begin{array}{l}\text { Transcriptional regulator, biotin repressor family } \\
\text { protein, NiaR }\end{array}$ & -2.7 \\
spd_1640 & Ribosyl nicotinamide transporter, PnuC-like, & 1.5 \\
spd_1824 & PnuC & \\
spd_1826 & Hypothetical protein & 3.5 \\
spd_1827 & NadC & 7.2 \\
\hline
\end{tabular}

Complete CDM contains $8 \mu \mathrm{M}$ of niacin.

a Gene numbers refer to D39 locus tags.

${ }^{b}$ D39 annotation/TIGR4 annotation (Lanie et al., 2007).

${ }^{c}$ Ratio represents the fold increase/decrease in the expression of genes in D39 $\Delta$ niaR compared to the D39 wild-type in complete CDM. Errors in the ratios never exceeded $10 \%$ of the given values.

from alanine, aspartate, and glutamate, and tryptophan metabolism into nicotinate D-ribonucleotide (Kanehisa et al., 2014).

\section{Niacin-dependent Expression of fba, rex, gapN, niaX, pncB, pnuC, gap, spd-1824, nadC, adhE, and adhB2}

Our niacin-dependent microarray data mentioned above indicated the role of niacin in the regulation of $f b a$, rex, gapN, niaX, pncB, pnuC, gap, spd-1824, nadC, adhE, and adhB2. To confirm our microarray results and further study the effect of niacin on the expression of $f b a, r e x, g a p N$, niaX, $p n c B$, pnuC, gap, spd-1824, nadC, adhE, and adhB2, we performed $\beta$-galactosidase assays with promoter lacZ-fusions of these genes constructed in S. pneumoniae D39 wild-type. Our $\beta$ galactosidase data demonstrated that the expression of $\mathrm{P} f b a-l a c Z$, Prex-lacZ, PgapN-lacZ, PniaX-lacZ, PpncB-lacZ, PpnuC-lacZ, Pgap-lacZ, PnadC-lacZ, PadhE-lacZ, and PadhB2-lacZ increased significantly in the absence of niacin in the medium (Figure 1). These data further confirm our microarray data described above and suggest the role of niacin in the regulation of these genes.

\section{Microarray Analysis of D39 $\Delta$ niaR}

Niacin genes are mostly regulated by a transcriptional regulator NiaR in different bacteria (Novichkov et al., 2010). In Firmicutes and Thermotogales, transcriptional regulator NiaR regulates the NAD biosynthesis and salvage of niacin (Rodionov et al., 2008a). NiaR was first studied in B. subtilis as a niacin-responsive transcriptional repressor that binds to its DNA targets in the presence of niacin (Rossolillo et al., 2005). NiaR belongs to a unique protein family, which possesses an N-terminal HTH (Helix-Turn-Helix) DNA binding domain (PF08279) and a $\mathrm{C}$-terminal effector binding domain, called the $3 \mathrm{H}$ domain (PF02829). S. pneumoniae also possesses a NiaR transcriptional regulator, which might be involved in the regulation of the niacin-regulated genes described above. Therefore, we decided to further study the role of NiaR in the regulation of these genes. 


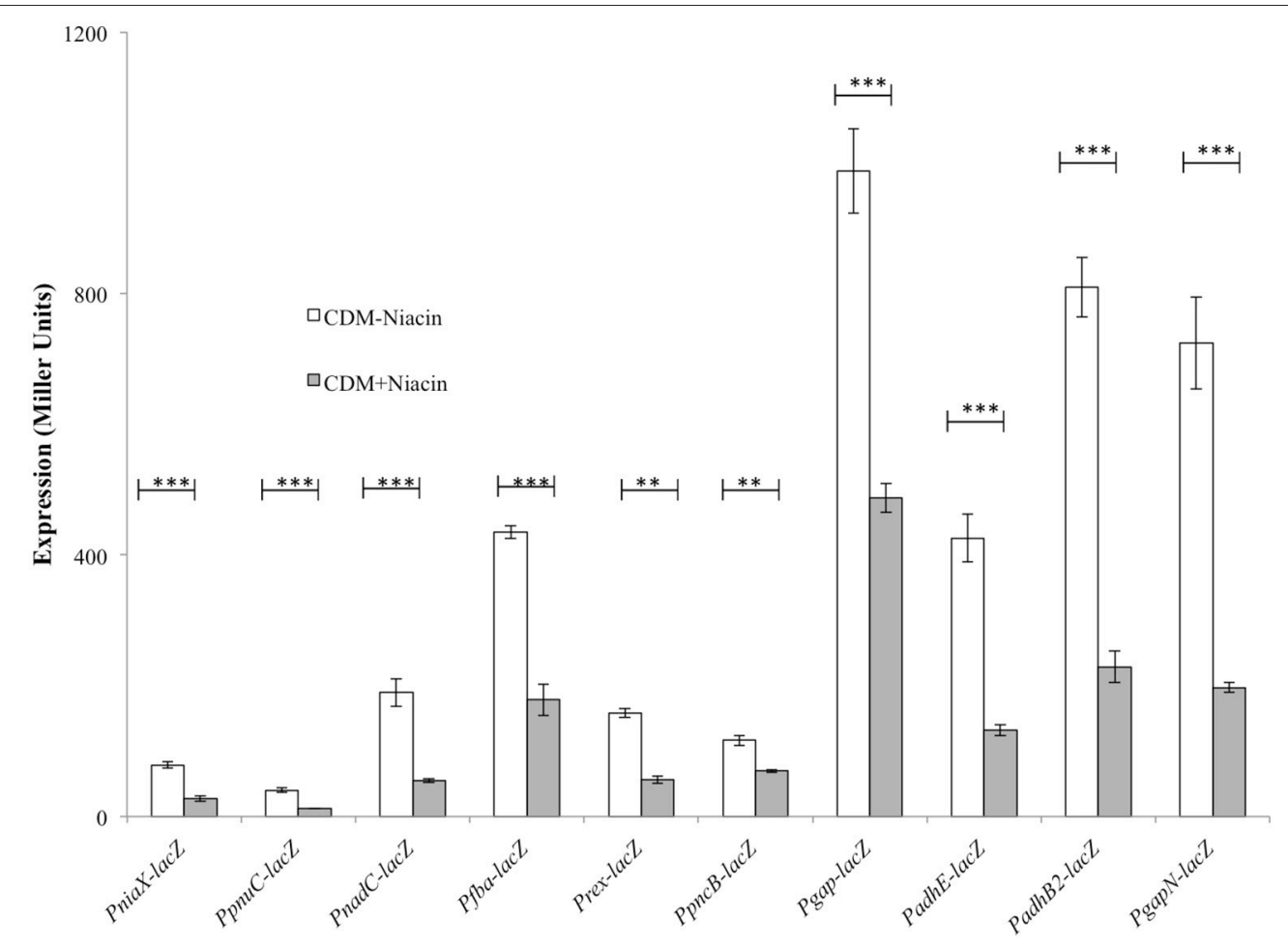

FIGURE 1 | Expression levels (in Miller units) of PniaX-lacZ, PpnuC-lacZ, PnadC-lacZ, Pfba-lacZ, Prex-lacZ, PpncB-lacZ, Pgap-lacZ, PadhE-lacZ, PadhB2-lacZ, and PgapN-lacZ in CDM with $\mathbf{0}$ and $\mathbf{1 0} \mathbf{~ m M ~ n i a c i n ~ i n ~ S . ~ p n e u m o n i a e ~ D 3 9 ~ w i l d - t y p e . ~ S t a n d a r d ~ d e v i a t i o n s ~ o f ~ t h r e e ~ i n d e p e n d e n t ~ e x p e r i m e n t s ~ a r e ~}$ indicated in bars. Statistical significance of the differences in the expression levels was determined by one-way ANOVA (NS, not significant, ${ }^{\star \star} P<0.001$, and ${ }^{\star \star \star} P<$ 0.0001).

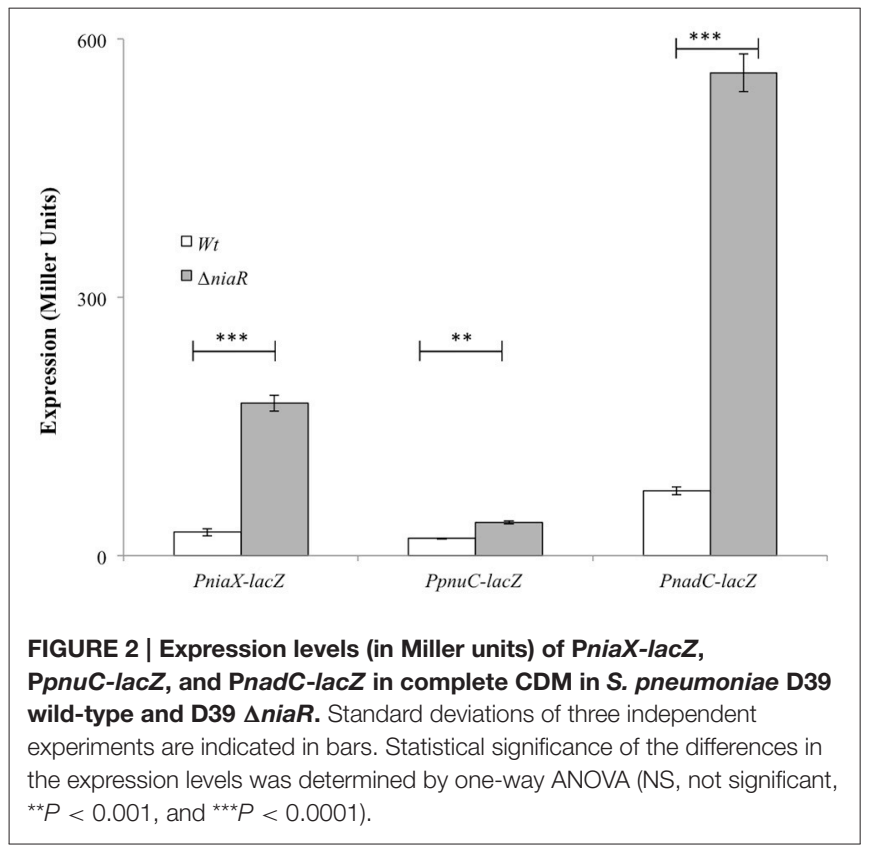

A deletion mutant of the niaR gene was constructed and microarray comparison of $S$. pneumoniae D39 $\triangle n i a R$ to $\mathrm{D} 39$ wild-type grown in complete CDM was performed to investigate the role of NiaR in S. pneumoniae D39. Complete CDM contains $8 \mu \mathrm{M}$ of niacin. Table 4 summarizes the transcriptome changes induced by the deletion of niaR in S. pneumoniae D39. Expression of niaR was downregulated about 3-fold confirming the niaR deletion in D39 $\triangle$ niaR. Expression of nadC, niaX, and pnuC was upregulated significantly in D39 $\Delta n i a R$, suggesting the role of $\mathrm{NiaR}$ as a transcriptional repressor of niaX, nadC, and pnuC in S. pneumoniae D39. Expression of spd-1824 and spd-1827 (coding for hypothetical proteins) was also upregulated. $S p d-1827$ is localized adjacent to nadC (spd-1826), but transcribed in opposite direction.

\section{Role of NiaR as a Transcriptional Repressor of niaX, nadC, and pnuC}

To further investigate the role of NiaR in the regulation of niaX, nadC, and pnuC, we transformed the lacZ-fusions of the promoter regions of niaX, nadC, and pnuC into D39 $\triangle$ niaR and performed $\beta$-galactosidase assays in complete CDM (Figure 2). The results of the $\beta$-galactosidase assays showed that the activity of all these promoters increased significantly in D39 $\triangle$ niaR compared to the D39 wild-type, confirming the role of NiaR as a transcriptional repressor of niaX, nadC, and pnuc. 


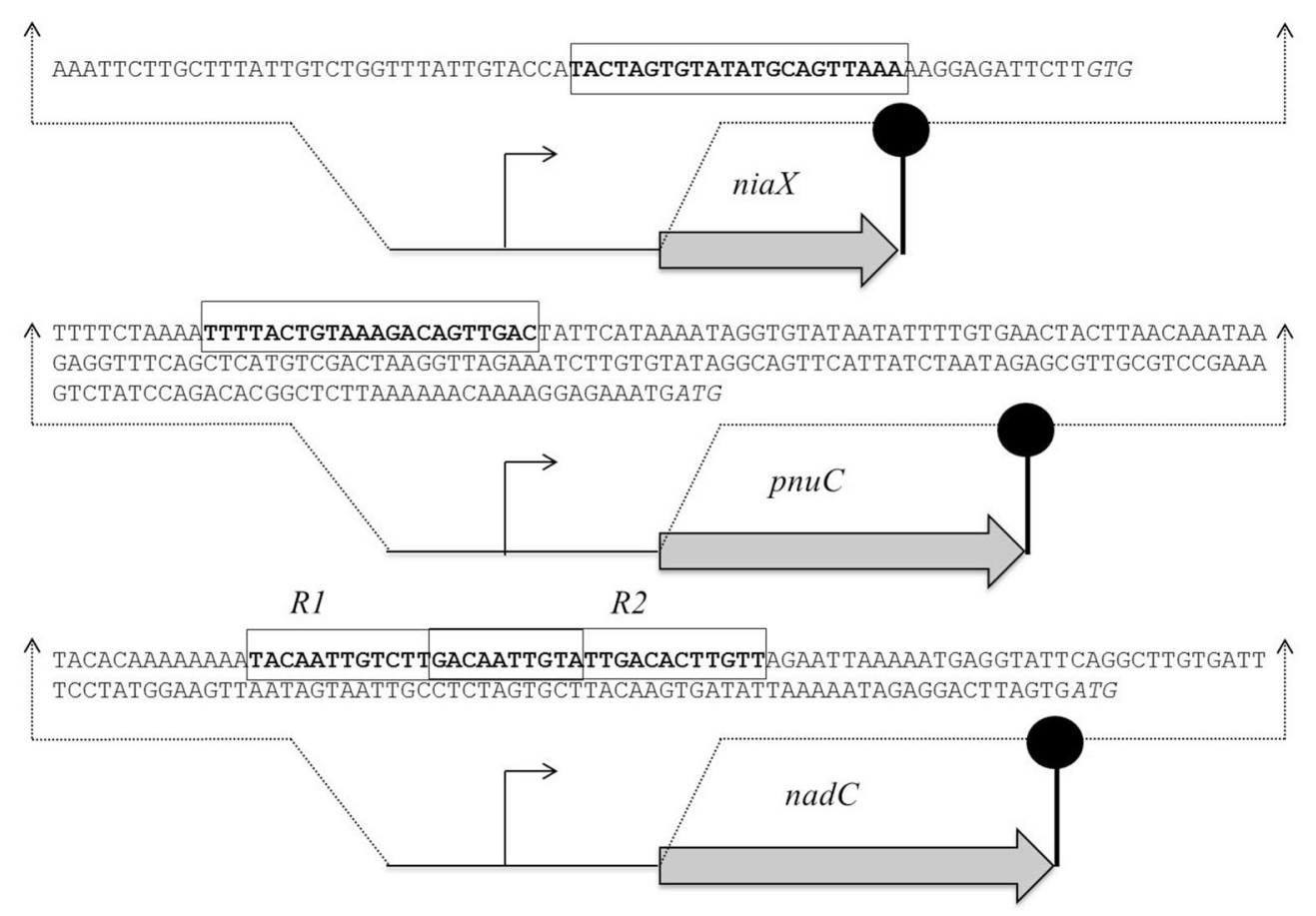

FIGURE 3 | Organization of the NiaR-regulated genes in S. pneumoniae D39. Putative NiaR operator sequences are rectangle and translational initiation sites are italicized, whereas the lollipop structures represent the putative transcriptional terminators. See text for further details.

\section{Prediction and Confirmation of the NiaR Site in PniaX, PnadC, and PpnuC}

The promoter regions of all of the upregulated genes, including spd_1824 and spd_1827, were analyzed by Genome2D software (Baerends et al., 2004) and a MEME motif sampler search (Bailey and Elkan, 1994). A 22-bp palindromic-like sequence was found in the promoter regions of niaX, nadC, and pnuC (Figure 3). This DNA sequence might serve as the NiaR operator site in S. pneumoniae. PniaX from different streptococci was also analyzed for the presence of NaiR site. The NiaR site present in the promoter region of niaX of different streptococci is shown in Figure 4. Weight matrix based on these putative NiaR sites ( $5^{\prime}$ - TACWRGTGTMTWKACASYTRWAW - $3^{\prime}$ ) was constructed (Figure 4).

The predicted NiaR operator site present in the promoter regions of niaX, nadC, and pnuC was further verified by promoter mutational experiment. For this purpose, we made transcriptional lacZ-fusions of PniaX, $\mathrm{P} p n u C$, and $\mathrm{P} n a d C$, where conserved bases in the putative NiaR sites were mutated in PniaX (5'- TACTAGTGTATATGCAGTTAAA- $3^{\prime}$ to $5^{\prime}$ - TACTAG

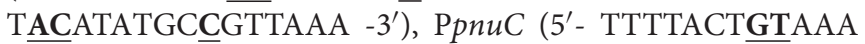
GACAGTTGAC $-3^{\prime}$ to $5^{\prime}$ - TTTTACTACAAAGACGGTTGAC -3'), PnadC-R1 (5'- TACAATTGTCTTGACAATTGTA $-3^{\prime}$ to $5^{\prime}$ - TACAATTACCTTGACCATTGTA - $\left.3^{\prime}\right)$, and PnadC-R2 (5'GACAATTGTATTGACACTTGTT $-3^{\prime}$ to $5^{\prime}$ - GACAATT $\underline{\text { ACAT }}$ TGACCCTTGTT $-3^{\prime}$ ). $\beta$-galactosidase assays were performed on cells grown in complete CDM. Complete CDM contains $8 \mu \mathrm{M}$ of niacin. The expression of PniaX and PpnuC with mutated conserved bases of NiaR operator sites increased significantly in
S. pneumoniae D39 wild-type, confirming that the predicted NiaR sites present in the promoter regions of niaX and $p n u C$ are active and intact in S. pneumoniae (Figure 5). Two putative operator sites for NiaR are present in PnadC (R1 and R2). We mutated both sites individually and performed $\beta$-galactosidase assays. We could only observe derepression (caused by NiaR) in the activity of PnadC when NiaR operator site 2 (R2) was mutated and did not observe any change in the activity of PnadC due to mutation in NiaR operator site 1 (R1) (Figure 5). These data suggest that operator site 2 (R2) is the functional operator site in PnadC.

\section{DISCUSSION}

NAD is an essential cofactor used by all living organisms. NAD synthesis is a tightly regulated intracellular process in bacteria (Huang et al., 2009). Bacteria acquire NAD in two main ways: through de novo synthesis and through the salvage pathway. Some bacteria do not have the ability to de novo synthesize $\mathrm{NAD}$ and must make use of the salvage pathway to import niacin or nicotinamide riboside through the substrate importers $\mathrm{NiaX}$ and PnuC, respectively. The de novo pathway synthesizes NAD from aspartic acid, whereas the salvage pathway brings intermediates many steps downstream into the NAD de novo synthesis pathway (Rodionov et al., 2008b). NiaX and PnuC are the two major importers in the NAD salvage pathway, where $\mathrm{NiaX}$ is responsible for niacin uptake, and PnuC transports nicotinamide riboside (Herbert et al., 2003; Sauer E. et al., 2004; Rodionov et al., 2008a, 2009). Our current study demonstrates the transcriptomic response of $S$. pneumoniae to niacin and 
A

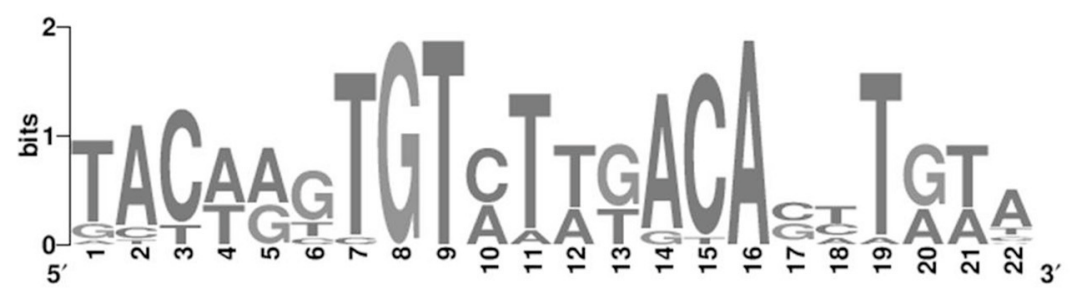

B

\begin{tabular}{|c|c|}
\hline СCTTGATTTATTGTCTTGTTTATTATACTATACTAGTGTATATACAGTTAAAAGGA-N007-ATG & -- Pniax \\
\hline GCAGGAATTTTATACATAGACAAATAACCTTACAGGTGTCTTTACACCTGTAAGGT-N031-ATG & PniaX \\
\hline ATAAATAGGAATCGGAACAGCGCATTGTTTTATAGGTGTCTTGACACTTGTAAAAC-N032-ATG & Pniax \\
\hline GAGCTTATGCTGTGGTTGTTTTTTTGATTTGCTAGGTGTCTTGACATCTGTCGCGT-N035-ATG & Pniax \\
\hline AGGCTTGCTTTTGAAGTCTAACTAAATAGTTACAGGTGTCTTGACACCTGTGCTTT-N165-ATG & Pniax \\
\hline TCTTGACTTGTTTGACAAAACAGTGTACTATACTACTGTATATACACATAAAGGAGATTATAATG & Pniax \\
\hline TGAGCAGGAGCATATCGTCTTTAATAATTAAACTAGTGTCTTGACAGATGTAAACA-N041-ATG & Pniax \\
\hline AACAAGGTATCGTTTAAAAATACCAAAAGATACAGGTGTCTTGATAGTTATTGGTT-N033-ATG & Pniax \\
\hline TCTTGACCTTTCTGCAAAAACAGTATACTATACTAGTGTATATACAGATAAAAAGG-N007-ATG & Pniax \\
\hline 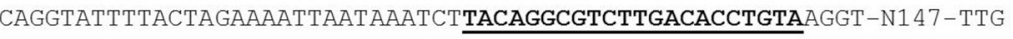 & Pniax \\
\hline TATTGACATATAGGTATATACAATGTAATATACTATTGTATTTACAGAAAAAGGAGAAGATTATG & Pniax \\
\hline TTGGTTTATTATATCACAGTTAAAAAACATTACAGGTGTCTTGACACCTGTAATGT-N043-ATG & Pniax \\
\hline GAAAGAGAAAATTTTCATGATTTTCTAAAATTTTACTGTAAAGACAGTTGACTATT-N161-ATG & $2 u$ \\
\hline $\begin{array}{l}\text { TTGGTACTTGACTATTATACACAAAAAAAATACAA } \\
\text { GAATTAAAAATGAGGTATTCAGGCTTGTGATTTCD }\end{array}$ & \\
\hline
\end{tabular}

FIGURE 4 | Identification of the NiaR operator site. (A) Weight matrix of the identified NiaR operator site in the promoter regions of niaX, nadC, and pnuC. (B) Position of the NiaR operator site in the promoter region of niaX, nadC, and pnuC in different streptococci. Putative NiaR operator sites are bold and underlined. SP, $S$. pneumoniae; SI, Streptococcus mitis; SA, Streptococcus agalactiae; SD, Streptococcus dysgalactiae; SE, Streptococcus equi; SG, Streptococcus gallolyticus; SN, Streptococcus gordonii; SM, Streptococcus mutans; SO, Streptococcus pyogenes; SS, Streptococcus sanguinis; SU, Streptococcus suis; ST, Streptococcus thermophiles; and SB, Streptococcus uberis.

reveals that a number of genes including $p n u C, p n c B$, and nadC are differentially expressed under the tested conditions. We further demonstrate that a transcriptional regulator NiaR acts as a transcriptional repressor of niaX, pnuC, and nadC in the presence of niacin.

An extracellular protein capable of modifying nicotinamide mononucleotide to an importable form appears to help NiaX and PnuC for importing nicotinamide mononucleotide or there may be another import system in S. pneumoniae (Johnson et al., 2015). There is significant variability between PnuC homologs (Jaehme et al., 2014), and the PnuC homologs from Haemophilus influenzae and Salmonella typhimurium do not import nicotinamide mononucleotide, but can transform it to an importable form for PnuC (nicotinamide riboside) through NadN or AphA, respectively (Kemmer et al., 2001; Grose et al., 2005). The PnuC proteins from H. influenzae, S. typhimurium, and $S$. pneumoniae all possess the motif for nicotinamide mononucleotide binding. Nevertheless, PnuC homologs from many other organisms lack the consensus binding residues (Kemmer et al., 2001; Sauer E. et al., 2004; Grose et al., 2005). These observations indicate that different groups of NAD salvage substrate importers (annotated as PnuC) import nicotinamide riboside and/or nicotinamide mononucleotide, and that NiaX imports niacin and/or nicotinamide mononucleotide as preferred substrates. Moreover, the amino acids in Salmonella PnuC curtailing import of nicotinamide mononucleotide are not conserved in the pneumococci, suggesting that the pneumococcal PnuC may permit this substrate along with nicotinamide riboside. Although, both $\mathrm{PnuC}$ and $\mathrm{NiaX}$ in S. pneumoniae may have acquired the ability to import nicotinamide mononucleotide, an extra importer (that is yet to be characterized) may also be present (Johnson et al., 2015). The role of PnuC in pneumococcal pathogenesis has been studied and PnuC could be a potential viable small molecule therapeutic target to halt disease progression in the host (Johnson et al., 2015). The proposed NAD pathway in S. pneumoniae states that niacin and nicotinamide enter the cells through NiaX, and PnuC transports nicotinamide riboside to the inside of the cell, whereas the transporter for nicotinamide mononucleotide is unknown (Johnson et al., 2015). spd-1411 encodes a nicotinamidase (PncA) that converts nicotinamide into niacin, which is further converted into nicotinate mononucleotide by a nicotinic acid phosphoribosyltransferase (PncB) (Johnson et al., 2015). The nicotinate mononucleotide is then converted into NAD by NadD and NadE. Moreover, NadD (nicotinate/nicotinamide nucleotide adenylyltransferase) converts nicotinamide riboside 


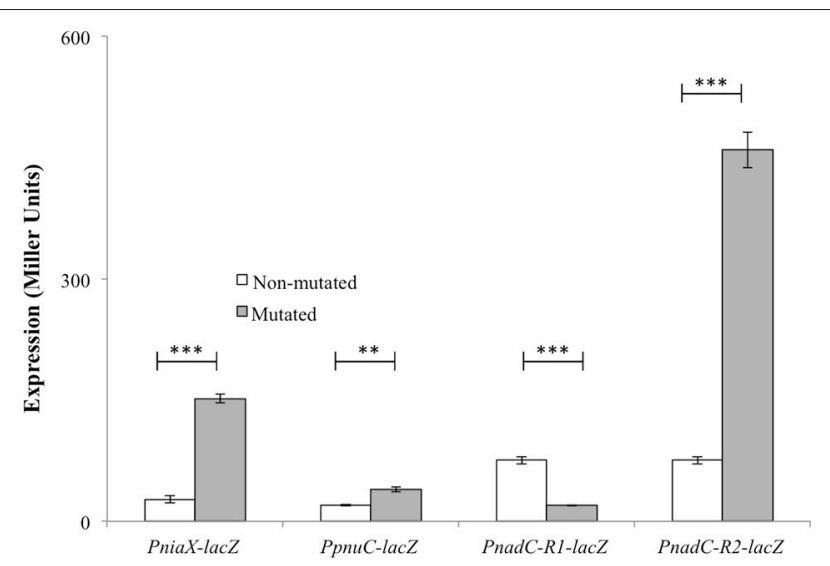

FIGURE 5 | Expression levels (in Miller units) of PniaX-lacZ, PpnuC-lacZ, and PnadC-lacZ with mutated and non-mutated NiaR operator sites in S. pneumoniae D39 wild-type grown in complete CDM. Standard deviations of three independent experiments are indicated in bars. Statistical significance of the differences in the expression levels was determined by one-way ANOVA (NS, not significant, ${ }^{\star \star} P<0.001$, and ${ }^{\star \star \star} P<$ 0.0001).

and nicotinamide mononucleotide into NAD (Johnson et al., 2015). Nicotinamide riboside augmentation has been attributed to several advantageous functions in the host, including shielding against mitochondrial myopathy (Khan et al., 2014), hearing loss (Brown et al., 2014) and obesity (Cantó et al., 2012). These functions may not be due to increasing NAD synthesis (Frederick et al., 2015), but may be due to overall bioavailability. While nicotinamide riboside is required for pathogen and host, luckily pneumococcal PnuC and its homologous in other bacteria do not have sequence homology to any proteins in the animal kingdom. Hence, PnuC could be a potential therapeutic target in bacterial species shielding this pathway without mammalian significance as has been effectively shown with $H$. influenzae (Sauer E. et al., 2004).

NiaR orthologs have been found in 30 out of 45 species from the Bacillus/Clostridium group (Firmicutes), in addition to the diverged groups of the Fusobacteria and Thermotogales and for the Bacillus/Clostrida group another DNA binding site was proposed (Rodionov et al., 2008a). There are two different types of DNA-binding sites of NiaR i.e., type I operator found in Firmicutes and Fusobacteria, and type II in the Thermotogales. The niacin-responsive transcription factor NiaR (known as YrxA in $B$. subtilis) was first recognized as a nicotinic acid-responsive repressor of the de novo NAD biosynthesis operon (nadABC) in B. subtilis (Rossolillo et al., 2005). NiaR regulation of the niacin salvage genes $p n c B$ (in Lactobacillus plantarum), pncA

\section{REFERENCES}

Afzal, M., Manzoor, I., and Kuipers, O. P. (2015). A fast and reliable pipeline for bacterial transcriptome analysis case study: serine-dependent gene regulation in Streptococcus pneumoniae. J. Vis. Exp. 98:e52649. doi: 10.3791/ 52649 (in Streptococcus pyogenes, Streptococcus equi, and Clostridium tetani), and/or the RNam salvage transporter pnuC (in $S$. pneumoniae and Streptococcus mutans) (Rodionov et al., 2008a) is less common. Moreover, the NiaR regulon contains membrane proteins that putatively have a role in niacin uptake. The most abundant NiaP family is found in ten NiaR-containing species (Bacilli, Lactobacilli and Thermotogales) in addition to several species that do not have the NiaR regulator (Rodionov et al., 2008a). Among Streptococci and Clostridia, NiaX is found in twelve genomes, and NiaY is found in five genomes (Bacilli and Clostridia). Several lines of genomic evidence support the putative involvement of these gene families in niacin uptake including the predicted co-regulation with NAD biosynthesis and niacin salvage genes, and co-occurrence with the niacin salvage genes $p n c B-p n c A$ (Rodionov et al., 2008a). Our study demonstrates that niaX, pnuC, and nadC are the genes that have a putative NiaR operator site in their promoter regions and are repressed by NiaR in the presence of niacin. We have further confirmed the NiaR operator sites in the promoter regions of niaX, pnuC, and nadC by mutagenesis studies. There are some other genes that are differentially expressed under our tested conditions (fba, rex, gapN, pncB-nadE, gap, spd-1824, spd-1827, $a d h E$, and $a d h B 2)$. The change in the expression of these genes suggests that these genes may have a role in the transport and biosynthesis of niacin or they may be upregulated due to some indirect effect of niacin genes. These genes do not have a putative NiaR operator site in their promoter regions suggesting the role of another transcriptional regulator in the regulation of fba, rex, gapN, pncB-nadE, gap, spd-1824, spd-1827, adhE, and $a d h B 2$. Therefore, we propose that the study of the regulatory mode of the above-mentioned genes would shed light on this possibility.

\section{AUTHOR CONTRIBUTIONS}

Substantial contributions to the conception or design of the work; or the acquisition, analysis, or interpretation of data for the work: MA, SS, and OK. Drafting the work or revising it critically for important intellectual content: MA, SS, and OK. Final approval of the version to be published: MA, SS, and OK. Agreement to be accountable for all aspects of the work in ensuring that questions related to the accuracy or integrity of any part of the work are appropriately investigated and resolved: MA, SS, and OK.

\section{ACKNOWLEDGMENTS}

MA is supported by the Government College University, Faisalabad, Pakistan under the faculty development program of HEC Pakistan. 
of bacterial transcriptome data. Genome Biol. 5:R37. doi: 10.1186/gb-20045-5-r37

Bailey, T. L., and Elkan, C. (1994). Fitting a mixture model by expectation maximization to discover motifs in biopolymers. Proc. Int. Conf. Intell. Syst. Mol. Biol. Biol. 2, 28-36.

Bogaert, D., De Groot, G. R., and Hermans, P. W. (2004). Streptococcus pneumoniae colonisation: the key to pneumococcal disease. Lancet Infect. Dis. 4, 144-154. doi: 10.1016/S1473-3099(04)00938-7

Bräsen, C., Esser, D., Rauch, B., and Siebers, B. (2014). Carbohydrate metabolism in Archaea: current insights into unusual enzymes and pathways and their regulation. Microbiol. Mol. Biol. Rev. 78, 89-175. doi: 10.1128/MMBR.0 0041-13

Brown, K. D., Maqsood, S., Huang, J.-Y., Pan, Y., Harkcom, W., Li, W., et al. (2014). Activation of SIRT3 by the $\mathrm{NAD}^{+}$precursor nicotinamide riboside protects from noise-induced hearing loss. Cell Metab. 20, 1059-1068. doi: 10.1016/j.cmet.2014.11.003

Cantó, C., Houtkooper, R. H., Pirinen, E., Youn, D. Y., Oosterveer, M. H., Cen, Y., et al. (2012). The $\mathrm{NAD}(+)$ precursor nicotinamide riboside enhances oxidative metabolism and protects against high-fat diet-induced obesity. Cell Metab. 15, 838-847. doi: 10.1016/j.cmet.2012.04.022

Chalkiadaki, A., and Guarente, L. (2012). Sirtuins mediate mammalian metabolic responses to nutrient availability. Nat. Rev. Endocrinol. 8, 287-296. doi: 10.1038/nrendo.2011.225

Chiarugi, A., Dölle, C., Felici, R., and Ziegler, M. (2012). The NAD metabolomea key determinant of cancer cell biology. Nat. Rev. Cancer 12, 741-752. doi: $10.1038 / \mathrm{nrc} 3340$

Cotter, P. A., and DiRita, V. J. (2000). Bacterial virulence gene regulation: an evolutionary perspective. Annu. Rev. Microbiol. 54, 519-565. doi: 10.1146/annurev.micro.54.1.519

Cummings, C. A., Bootsma, H. J., Relman, D. A., and Miller, J. F. (2006). Speciesand strain-specific control of a complex, flexible regulon by Bordetella BvgAS. J. Bacteriol. 188, 1775-1785. doi: 10.1128/JB.188.5.1775-1785.2006

Edwards, R. L., Bryan, A., Jules, M., Harada, K., Buchrieser, C., and Swanson, M. S. (2013). Nicotinic acid modulates Legionella pneumophila gene expression and induces virulence traits. Infect. Immun. 81, 945-955. doi: 10.1128/IAI.00 999-12

Eguchi, Y., Oshima, T., Mori, H., Aono, R., Yamamoto, K., Ishihama, A., et al. (2003). Transcriptional regulation of drug efflux genes by EvgAS, a two-component system in Escherichia coli. Microbiology 149, 2819-2828. doi: 10.1099/mic.0.26460-0

Frederick, D. W., Davis, J. G., Dávila, A., Agarwal, B., Michan, S., Puchowicz, M. A., et al. (2015). Increasing NAD synthesis in muscle via nicotinamide phosphoribosyltransferase is not sufficient to promote oxidative metabolism. J. Biol. Chem. 290, 1546-1558. doi: 10.1074/jbc.M114.579565

Grose, J. H., Bergthorsson, U., Xu, Y., Sterneckert, J., Khodaverdian, B., and Roth, J. R. (2005). Assimilation of nicotinamide mononucleotide requires periplasmic AphA phosphatase in Salmonella enterica. J. Bacteriol. 187, 4521-4530. doi: 10.1128/JB.187.13.4521-4530.2005

Halfmann, A., Hakenbeck, R., and Bruckner, R. (2007). A new integrative reporter plasmid for Streptococcus pneumoniae. FEMS Microbiol. Lett. 268, 217-224. doi: $10.1111 / j .1574-6968.2006 .00584 . x$

Herbert, M., Sauer, E., Smethurst, G., Kraiss, A., Hilpert, A.-K., and Reidl, J. (2003). Nicotinamide ribosyl uptake mutants in Haemophilus influenzae. Infect. Immun. 71, 5398-5401. doi: 10.1128/IAI.71.9.5398-5401.2003

Huang, N., De Ingeniis, J., Galeazzi, L., Mancini, C., Korostelev, Y. D., Rakhmaninova, A. B., et al. (2009). Structure and function of an ADPribose-dependent transcriptional regulator of NAD metabolism. Structure 17, 939-951. doi: 10.1016/j.str.2009.05.012

Ishino, Y., Shinagawa, H., Makino, K., Tsunasawa, S., Sakiyama, F., and Nakata, A. (1986). Nucleotide sequence of the lig gene and primary structure of DNA ligase of Escherichia coli. Mol. Gen. Genet. 204, 1-7. doi: 10.1007/BF00330179

Israelsen, H., Madsen, S. M., Vrang, A., Hansen, E. B., and Johansen, E. (1995). Cloning and partial characterization of regulated promoters from Lactococcus lactis Tn917-lacZ integrants with the new promoter probe vector, pAK80. Appl. Environ. Microbiol. 61, 2540-2547.

Jaehme, M., Guskov, A., and Slotboom, D. J. (2014). Crystal structure of the vitamin B3 transporter PnuC, a full-length SWEET homolog. Nat. Struct. Mol. Biol. 21, 1013-1015. doi: 10.1038/nsmb.2909
Johnson, M. D. L., Echlin, H., Dao, T. H., and Rosch, J. W. (2015). Characterization of NAD salvage pathways and their role in virulence in Streptococcus pneumoniae. Microbiology 161, 2127-2136. doi: 10.1099/mic.0.000164

Jurtshuk, P. (1996). "Bacterial Metabolism," in Medical Microbiology, ed S. Baron (Galveston, TX: University of Texas Medical Branch at Galveston).

Kadioglu, A., Weiser, J. N., Paton, J. C., and Andrew, P. W. (2008). The role of Streptococcus pneumoniae virulence factors in host respiratory colonization and disease. Nat. Rev. Microbiol. 6, 288-301. doi: 10.1038/nrmicro1871

Kanehisa, M., Goto, S., Sato, Y., Kawashima, M., Furumichi, M., and Tanabe, M. (2014). Data, information, knowledge and principle: back to metabolism in KEGG. Nucleic Acids Res. 42, D199-D205. doi: 10.1093/nar/gkt1076

Kemmer, G., Reilly, T. J., Schmidt-Brauns, J., Zlotnik, G. W., Green, B. A., Fiske, M. J., et al. (2001). NadN and e (P4) are essential for utilization of NAD and nicotinamide mononucleotide but not nicotinamide riboside in Haemophilus influenzae. J. Bacteriol. 183, 3974-3981. doi: 10.1128/JB.183.13.3974-3981.2001

Khan, N. A., Auranen, M., Paetau, I., Pirinen, E., Euro, L., Forsström, S., et al. (2014). Effective treatment of mitochondrial myopathy by nicotinamide riboside, a vitamin B3. EMBO Mol. Med. 6, 721-731. doi: $10.1002 / \mathrm{emmm} .201403943$

Kloosterman, T. G., Bijlsma, J. J. E., Kok, J., and Kuipers, O. P. (2006). To have neighbour's fare: extending the molecular toolbox for Streptococcus pneumoniae. Microbiology 152, 351-359. doi: 10.1099/mic.0.28521-0

Kotrbova-Kozak, A., Kotrba, P., Inui, M., Sajdok, J., and Yukawa, H. (2007). Transcriptionally regulated adhA gene encodes alcohol dehydrogenase required for ethanol and n-propanol utilization in Corynebacterium glutamicum R. Appl. Microbiol. Biotechnol. 76, 1347-1356. doi: 10.1007/s00253-007-1094-6

Lanie, J. A., Ng, W. L., Kazmierczak, K. M., Andrzejewski, T. M., Davidsen, T. M., Wayne, K. J., et al. (2007). Genome sequence of Avery's virulent serotype 2 strain D39 of Streptococcus pneumoniae and comparison with that of unencapsulated laboratory strain R6. J. Bacteriol. 189, 38-51. doi: 10.1128/JB.01148-06

Luong, T. T., Kim, E.-H., Bak, J. P., Nguyen, C. T., Choi, S., Briles, D. E., et al. (2015). Ethanol-induced alcohol dehydrogenase E (AdhE) potentiates pneumolysin in Streptococcus pneumoniae. Infect. Immun. 83, 108-119. doi: 10.1128/IAI.02434-14

Masuda, N., and Church, G. M. (2002). Escherichia coli gene expression responsive to levels of the response regulator EvgA. J. Bacteriol. 184, 6225-6234. doi: 10.1128/JB.184.22.6225-6234.2002

Masuda, N., and Church, G. M. (2003). Regulatory network of acid resistance genes in Escherichia coli. Mol. Microbiol. 48, 699-712. doi: 10.1046/j.1365-2958.2003.03477.x

Matsubara, K., Yokooji, Y., Atomi, H., and Imanaka, T. (2011). Biochemical and genetic characterization of the three metabolic routes in Thermococcus kodakarensis linking glyceraldehyde 3-phosphate and 3-phosphoglycerate. Mol. Microbiol. 81, 1300-1312. doi: 10.1111/j.1365-2958.2011.07762.x

McPheat, W. L., Wardlaw, A. C., and Novotny, P. (1983). Modulation of Bordetella pertussis by nicotinic acid. Infect. Immun. 41, 516-522.

Miller, J. F., Roy, C. R., and Falkow, S. (1989). Analysis of Bordetella pertussis virulence gene regulation by use of transcriptional fusions in Escherichia coli. J. Bacteriol. 171, 6345-6348. doi: 10.1128/jb.171.11.6345-6348.1989

Neves, A. R., Ventura, R., Mansour, N., Shearman, C., Gasson, M. J., Maycock, C., et al. (2002). Is the glycolytic flux in Lactococcus lactis primarily controlled by the redox charge? Kinetics of NAD $(+)$ and NADH pools determined in vivo by 13C NMR. J. Biol. Chem. 277, 28088-28098. doi: 10.1074/jbc.M202573200

Nishino, K., Inazumi, Y., and Yamaguchi, A. (2003). Global analysis of genes regulated by EvgA of the two-component regulatory system in Escherichia coli. J. Bacteriol. 185, 2667-2672. doi: 10.1128/JB.185.8.2667-2672.2003

Nobelmann, B., and Lengeler, J. W. (1996). Molecular analysis of the gat genes from Escherichia coli and of their roles in galactitol transport and metabolism. J. Bacteriol. 178, 6790-6795. doi: 10.1128/jb.178.23.6790-6795.1996

Novichkov, P. S., Laikova, O. N., Novichkova, E. S., Gelfand, M. S., Arkin, A. P., Dubchak, I., et al. (2010). RegPrecise: a database of curated genomic inferences of transcriptional regulatory interactions in prokaryotes. Nucleic Acids Res. 38, D111-D118. doi: 10.1093/nar/gkp894

Patel, M. S., Nemeria, N. S., Furey, W., and Jordan, F. (2014). The pyruvate dehydrogenase complexes: structure-based function and regulation. J. Biol. Chem. 289, 16615-16623. doi: 10.1074/jbc.R114.563148 
Phillips, N. J., John, C. M., Reinders, L. G., Gibson, B. W., Apicella, M. A., and Griffiss, J. M. (1990). Structural models for the cell surface lipooligosaccharides of Neisseria gonorrhoeae and Haemophilus influenzae. Biomed. Environ. Mass Spectrom. 19, 731-745. doi: 10.1002/bms.1200191112

Rodionov, D. A., De Ingeniis, J., Mancini, C., Cimadamore, F., Zhang, H., Osterman, A. L., et al. (2008b). Transcriptional regulation of NAD metabolism in bacteria: NrtR family of Nudix-related regulators. Nucleic Acids Res. 36, 2047-2059. doi: 10.1093/nar/gkn047

Rodionov, D. A., Hebbeln, P., Eudes, A., ter Beek, J., Rodionova, I. A., Erkens, G. B., et al. (2009). A novel class of modular transporters for vitamins in prokaryotes. J. Bacteriol. 191, 42-51. doi: 10.1128/JB.01208-08

Rodionov, D. A., Li, X., Rodionova, I. A., Yang, C., Sorci, L., Dervyn, E., et al. (2008a). Transcriptional regulation of NAD metabolism in bacteria: genomic reconstruction of NiaR (YrxA) regulon. Nucleic Acids Res. 36, 2032-2046. doi: 10.1093/nar/gkn046

Rossolillo, P., Marinoni, I., Galli, E., Colosimo, A., and Albertini, A. M. (2005). YrxA is the transcriptional regulator that represses de novo NAD biosynthesis in Bacillus subtilis. J. Bacteriol. 187, 7155-7160. doi: 10.1128/JB.187.20.7155-7160.2005

Satoh, M. S., and Lindahl, T. (1992). Role of poly(ADP-ribose) formation in DNA repair. Nature 356, 356-358. doi: 10.1038/356356a0

Sauer, E., Merdanovic, M., Mortimer, A. P., Bringmann, G., and Reidl, J. (2004). $\mathrm{PnuC}$ and the utilization of the nicotinamide riboside analog 3-aminopyridine in Haemophilus influenzae. Antimicrob. Agents Chemother. 48, 4532-4541. doi: 10.1128/AAC.48.12.4532-4541.2004

Sauer, U., Canonaco, F., Heri, S., Perrenoud, A., and Fischer, E. (2004). The soluble and membrane-bound transhydrogenases $\mathrm{UdhA}$ and PntAB have divergent functions in NADPH metabolism of Escherichia coli. J. Biol. Chem. 279, 6613-6619. doi: 10.1074/jbc.M311657200
Schneider, D. R., and Parker, C. D. (1982). Effect of pyridines on phenotypic properties of Bordetella pertussis. Infect. Immun. 38, 548-553.

Shafeeq, S., Afzal, M., Henriques-Normark, B., and Kuipers, O. P. (2015). Transcriptional profiling of UlaR-regulated genes in Streptococcus pneumoniae. Genomics Data 4, 57-59. doi: 10.1016/j.gdata.2015.02.004

Sirover, M. A. (2011). On the functional diversity of glyceraldehyde-3phosphate dehydrogenase: biochemical mechanisms and regulatory control. Biochim. Biophys. Acta 1810, 741-751. doi: 10.1016/j.bbagen.2011. 05.010

Titgemeyer, F., and Hillen, W. (2002). Global control of sugar metabolism: a gram-positive solution. Antonie Van Leeuwenhoek 82, 59-71. doi: 10.1023/A:1020628909429

Wei, Z., Fu, Y., Zhou, E., Tian, Y., Yao, M., Li, Y., et al. (2014). Effects of niacin on Staphylococcus aureus internalization into bovine mammary epithelial cells by modulating NF- $\kappa$ B activation. Microb. Pathog. 71-72, 62-67. doi: 10.1016/j.micpath.2014.03.005

Wilkinson, A., Day, J., and Bowater, R. (2001). Bacterial DNA ligases. Mol. Microbiol. 40, 1241-1248. doi: 10.1046/j.1365-2958.2001.02479.x

Conflict of Interest Statement: The authors declare that the research was conducted in the absence of any commercial or financial relationships that could be construed as a potential conflict of interest.

Copyright (c) 2017 Afzal, Kuipers and Shafeeq. This is an open-access article distributed under the terms of the Creative Commons Attribution License (CC BY). The use, distribution or reproduction in other forums is permitted, provided the original author(s) or licensor are credited and that the original publication in this journal is cited, in accordance with accepted academic practice. No use, distribution or reproduction is permitted which does not comply with these terms. 